\title{
A Novel Step Counting Algorithm Based on Acceleration and Gravity Sensors of a Smart-Phone
}

\author{
Qingchi Zeng, Biao Zhou, Changqiang Jing, Nammoon Kim and Youngok Kim \\ Department of Electronic Engineering, Kwangwoon University, 447-1Wol gye- \\ dong, No won-gu, Seoul, 139-701, Republic of Korea \\ kimyoungok@kw.ac.kr
}

\begin{abstract}
Various sensors have been recently integrated in a smart-phone and step detection method based on the acceleration sensor of a smart-phone has been introduced for indoor positioning scheme. Many researchers have put their interests on the study of step detection algorithm based on the acceleration sensor. However, the estimation performances of these methods are not good enough or just suitable for the situation when the position of the smart-phone is fixed in hand because the performance is significantly degraded in other cases. In this paper, a novel step counting algorithm based on the acceleration and the gravity sensors is proposed to enhance the estimation performance regardless of the position of a smart-phone and the motions of a pedestrian likewise walking or running. The effectiveness of the proposed scheme is demonstrated with experiments and the performance of the proposed scheme is compared with those of the conventional schemes. According to the results, the performance of the proposed scheme is enhanced compared to the conventional schemes no matter what the pedestrian is walking or running although the phone is in different places like trouser pocket, shirt pocket and hands.
\end{abstract}

Keywords: Indoor positioning, acceleration sensor, gravity sensor, step counting, dead reckoning

\section{Introduction}

It is well known that the performance of Global Positioning System (GPS) is not good enough for finding the position of objects in the indoor environments. Since the object, especially a person, can go anywhere regardless of indoor or outdoor, the positioning scheme has been actively studied by researchers in these years. The indoor positioning scheme is usually based on several techniques, such as wireless signal, optical signal, and ultrasonic signal receiving techniques. However, all the signal receiving techniques must have a number of access points (APs), which transmit/receive the signals to calculate the coordinates of the target object [1]-[4]. It means that a sufficient number of APs is required to achieve effective accuracy of position estimation and even more APs is needed to improve the performance of position estimation.

Dead Reckoning (DR) is a technique that estimates the position of the pedestrian using motion sensors. Pedestrian Dead Reckoning (PDR) also is well known as one of indoor positioning techniques. The PDR technique determines the latest position of a pedestrian by adding an estimated displacement to the known previous position [8]. In the PDR scheme, step detection is an important part to estimate the displacement. After the step is detected, the step counting is done to find the numbers of steps of a pedestrian while he/she is walking or running. The basic concept of step detection is to transform the raw date of acceleration sensor to step 
parameters. At the beginning, sensors were attached to shoes or clothes to collect the acceleration data. Owe to the development of technology, many kinds of sensors have been recently integrated in a smart-phone and various step detection methods based on the acceleration sensor of a smart-phone are introduced by researchers. However, the estimation performances of these methods are not good enough or just suitable for the situation when the position of the smart-phone is fixed in hand because the performance is significantly degraded in other cases.

In this paper, a novel step counting algorithm based on the acceleration and the gravity sensors is proposed to enhance the estimation performance regardless of the position of a smart-phone and the motions of a pedestrian likewise walking or running. The effectiveness of the proposed scheme is demonstrated with experiments and the performance of the proposed scheme is compared with those of the conventional schemes. According to the results, the performance of the proposed scheme is enhanced compared to the conventional schemes no matter what the pedestrian is walking or running although the phone is in different places like trouser pocket, shirt pocket and hands. Therefore, it is worth to note that the proposed scheme is a concise and effective way to count the steps on real time.

The rest of paper is organized as follows. Section 2 represents the related works of conventional step counting methods. In Section 3, the detailed description of the proposed scheme is given. In Section 4, the performance of the proposed scheme is evaluated with experiments and compared with the conventional scheme. Finally, the concluding remarks are given in Section 5.

\section{Related Works}

In recent years, many researchers have put their interests on the study of step detection algorithm based on the acceleration sensor. In the conventional step detection algorithms, there are three common methods in processing of acceleration data: 1) using acceleration data along vertical orientation (Z-axis), 2) the Root Mean Square (RMS) of three axes, and 3) using the rotation matrices to transform the acceleration data from device frame to earth frame (Acceleration \& Matrices).

A PDR system with a phone location-awareness algorithm is introduced in [9]. In the paper, the accelerometer is attached to the user's waist belt and the authors only put attention to the analysis of the acceleration data on the $\mathrm{Z}$ axis of the accelerometer. The disadvantage of their method is that the acceleration data is disordered when the orientation of the accelerometer is changed.

In [10] and [11], a three-axis accelerometer is used to collect the acceleration data along each axis. In order to analyze the feature of the step, the RMS concept is used in processing the raw acceleration data as follows:

$$
R M S=\sqrt{x^{2}+y^{2}+z^{2}}
$$

where $\mathrm{x}, \mathrm{y}$ and $\mathrm{z}$ are the acceleration data of the $\mathrm{x}-, \mathrm{y}$ - and $\mathrm{z}$-axis. However, the acceleration sensor does not always keep on a steady state while the user is walking or running, thus noise tends to be increased as the moving speed of the user is increased.

In [11], a rotation matrix is used to transform the acceleration data of the device from device frame to earth frame. This method just focuses on the acceleration data along the vertical orientation likewise method 1). Compared with the method 1), the advantage of this method is that it can be used in a non-steady state during the movement of the user. Although the gesture of the accelerometer is changed, the data along the vertical orientation maintains normal. However, the disadvantage of this method is similar with the method 2), i.e., it is valid for only low speed of movement.

In the following section, a novel step counting algorithm based on the acceleration and the gravity sensors is proposed and its performance is compared with those of the above conventional schemes. In this paper, s1, s2, s3 are used to 
represent method 1), method 2) and method 3) respectively as discussed above, and s4 is used to represent the proposed method.

\section{Description of Proposed Scheme}

In this section, the proposed step counting method is described. In part A, the data collecting process under considering the characteristics of Android sensors is presented. The raw data processing and the new proposed data processing methods are described in Part B. In Part C, the proposed step detection method is described.

\subsection{Data Collecting Process}

As one of the most famous open source platform, the Android system supports many kinds of motion sensors. For experiments, an application based on the Android system is developed for data collecting and step detection. With the developed application, the experiment results are saved in SD-card and the step numbers are shown in the screen of the smart-phone in real time.

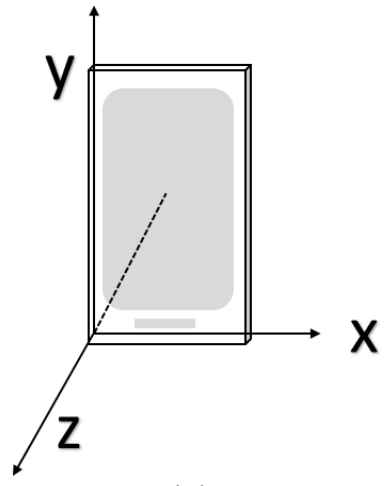

(a)

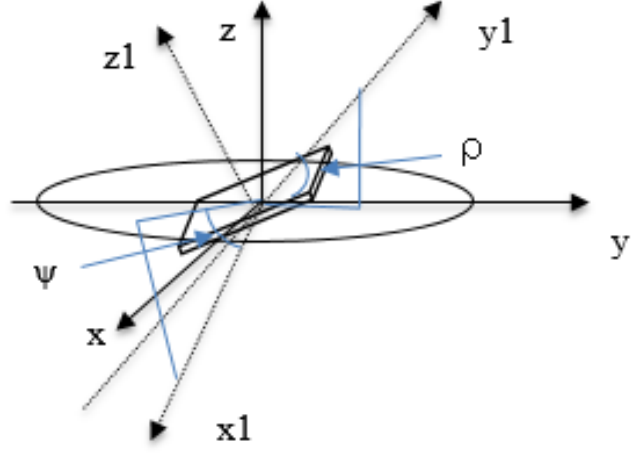

(b)

\section{Figure 1. (a) Sensor Coordinate System (b) Angle $\rho$ Describe Angle between $y 1$ axis and the Plane, and Angle $\Psi$ Describe the Angle between x1 axis and the Plane}

The proposed step counting algorithm is based on the acceleration and the gravity sensors of a smart-phone. The acceleration sensor measures the acceleration, which is applied to the device by the movement of the user, including the force of gravity. The built-in acceleration sensor in the smart-phone is a tri-axis accelerometer, which provides the acceleration measurements in $\mathrm{m} / \mathrm{s}^{2}$ along each of $\mathrm{X}, \mathrm{Y}, \mathrm{Z}$ axes [12]. As shown in Figure 1 (a), the coordinate system of the acceleration sensor is defined with the screen of the phone and its default orientation.

The gravity sensor provides a three dimensional vector data indicating the direction and the magnitude of gravity. The gravity sensor is a software-based sensor. Software-based sensors are not physical devices and derive their data from one or more of the hardware-based sensors and are sometimes called virtual sensors or synthetic sensors. Actually, gravity sensor data can be isolated from gravity by using a low-pass filter. The units are the same as those used by the acceleration sensor $\left(\mathrm{m} / \mathrm{s}^{2}\right)$, and the coordinate system is the same as the one used by the acceleration sensor. When the smart-phone is in a steady state, the readings of the acceleration sensor and the gravity sensor are almost same. However, when the 
smart-phone is in state of motion, the readings of these two sensors are significantly different. While the user with the smart-phone is moving, therefore, the acceleration sensor can be used for measuring acceleration data and the gravity sensor can be used to recognize the gesture of the smart-phone.

It is known that each gravity component of gravity sensor can be observed along the three axes [13]. Once the smart-phone is fixed in a stable state, the rotation around the $\mathrm{X}$ axis and the rotation around the $\mathrm{Y}$ axis can be detected by reading the gravity sensor. Note that the rotations are described in Figure 1 (b). The relationship between the gravity data and the rotation angle can be expressed as follows [13]:

$$
\begin{aligned}
& \psi=\arctan \left(\frac{g_{x}}{\sqrt{g_{y}^{2}+g_{z}^{2}}}\right) \\
& \rho=\arctan \left(\frac{g_{y}}{\sqrt{g_{x}^{2}+g_{z}^{2}}}\right)
\end{aligned}
$$

where $g_{x}, g_{y}$ and $g_{z}$ represent the gravity sensor parameters along three different axes.

In [14], it is shown that the accuracy of the equations (2) and (3) is almost constant inside the whole range of values that $\rho$ and $\psi$ can be assumed. Note that the value along the axis is close to 9.8 , when one axis approaches the vertical orientation.

In the conventional methods, an accelerometer is widely used to collect the acceleration data. As the moving speed of the accelerometer becomes faster, however, the accuracy of the step detection based on the acceleration sensor is degraded significantly. Besides this, when the gesture of smart-phone is changed, the acceleration data also is changed significantly. To overcome these disadvantages, the acceleration sensor as well as the gravity sensor is used in the proposed scheme.

\subsection{Raw Data Process}

In this section, the detailed data processing scheme with the raw data from the accelerometer sensor and the gravity sensor is discussed. By using the proposed scheme, no matter where the smart-phone is placed, such as holding in hand, putting in trouser pocket, shirt pocket or just in handbag, and no matter what the user walks or runs, the performance of step counting becomes always reliable.

As discussed in the previous section, when the gesture of the smart-phone is changed, the gravity sensor data is changed. In other words, we can recognize the gesture of smart-phone based on the raw data of gravity senor. In the proposed scheme, a combination value, $s$, of datum from the gravity sensor and the acceleration sensor is defined as follows:

$$
s=g_{x} a_{x}+g_{y} a_{y}+g_{z} a_{z}
$$

where $\mathrm{a}_{\mathrm{x}}, \mathrm{a}_{\mathrm{y}}$ and $\mathrm{a}_{\mathrm{z}}$ represent the acceleration parameters along $\mathrm{X}, \mathrm{Y}$ and $\mathrm{Y}$ axes, respectively, while $\mathrm{g}_{\mathrm{x}}, \mathrm{g}_{\mathrm{y}}$ and $\mathrm{g}_{\mathrm{z}}$ represent the gravity sensor parameters along three different axes. As described in the above, $g_{x}, g_{y}$ and $g_{z}$ are the gravity components along the three axes. Thus, the relationship between the components and the gravity acceleration value, $g$, can be expressed as follows:

$$
g_{x}^{2}+g_{y}^{2}+g_{z}^{2}=g^{2}
$$

Note that the constant gravity acceleration value, $g$, representing the gravity acceleration of the earth is equal to $9.8 \mathrm{~m} / \mathrm{s}^{2}$ in most places. 
Here the ratio of coefficient (ROC) also is defined to show the weight of each axis and it is expressed as follows:

$$
\begin{aligned}
R_{x} & =\frac{\left|g_{x}\right|}{\left|g_{x}\right|+\left|g_{y}\right|+\left|g_{z}\right|} \\
R_{y} & =\frac{\left|g_{y}\right|}{\left|g_{x}\right|+\left|g_{y}\right|+\left|g_{z}\right|} \\
R_{z} & =\frac{\left|g_{z}\right|}{\left|g_{x}\right|+\left|g_{y}\right|+\left|g_{z}\right|}
\end{aligned}
$$

Figure 2 shows the change of each axis when the smart-phone is rotated. As described in the figure, when the smart-phone rotates around an $\mathrm{X}$ axis, the modulus of $\mathrm{g}_{\mathrm{y}}$ becomes larger, while the modulus of $\mathrm{g}_{\mathrm{z}}$ becomes smaller. Note that the normalized changed-values of the gravity component along the three axes are described with the ROC in the figure. At first, the phone is parallel to the ground, and then start to rotate around $\mathrm{X}$ axis. When the $\mathrm{Y}$ axis is vertical to the ground, the ratio of the $\mathrm{y}$ axis is the largest. After this, when the phone is parallel to the ground again, the ratio of $\mathrm{z}$ axis is the largest.

While the user is walking, the situation of the changing ratio is more complex as shown in Figure 3. As shown in the figure, the ratio of each axis continuously changes as the orientation is changed. Based on this observation, therefore, auto calibration coefficients, which are adjusted to the ideal ratio, can be applied for raw data processing.

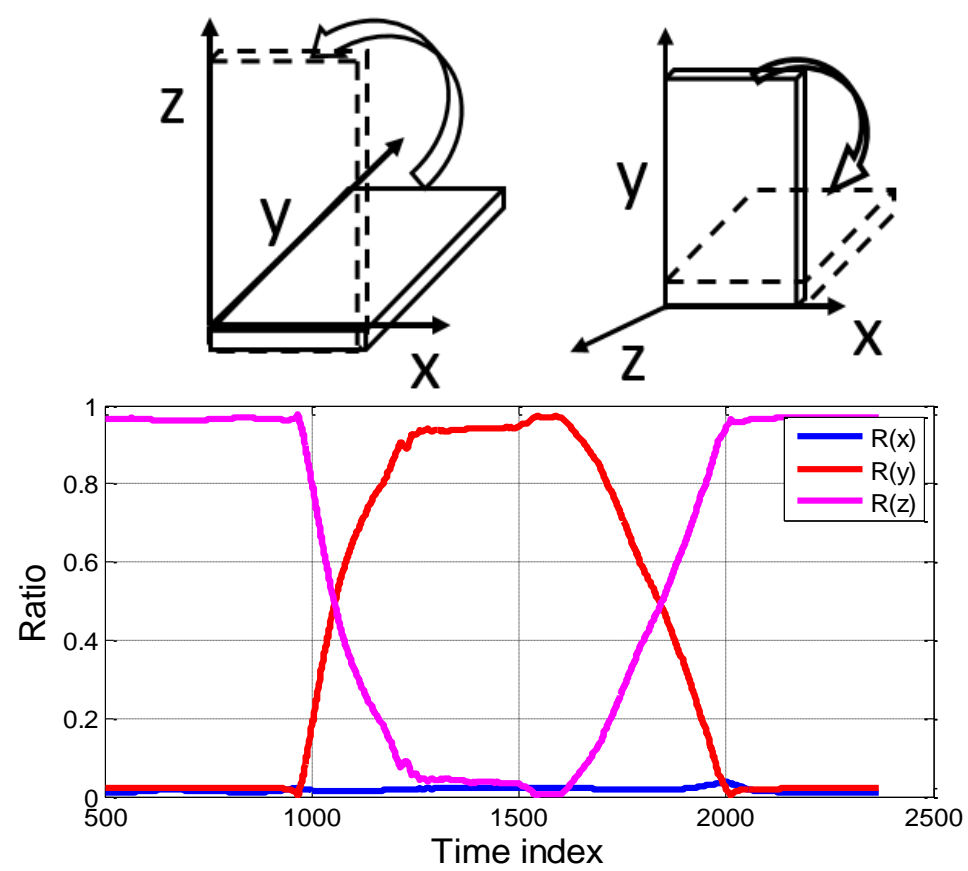

Figure 2. The Ratio Change when the Phone is Rotating Around X Axis 

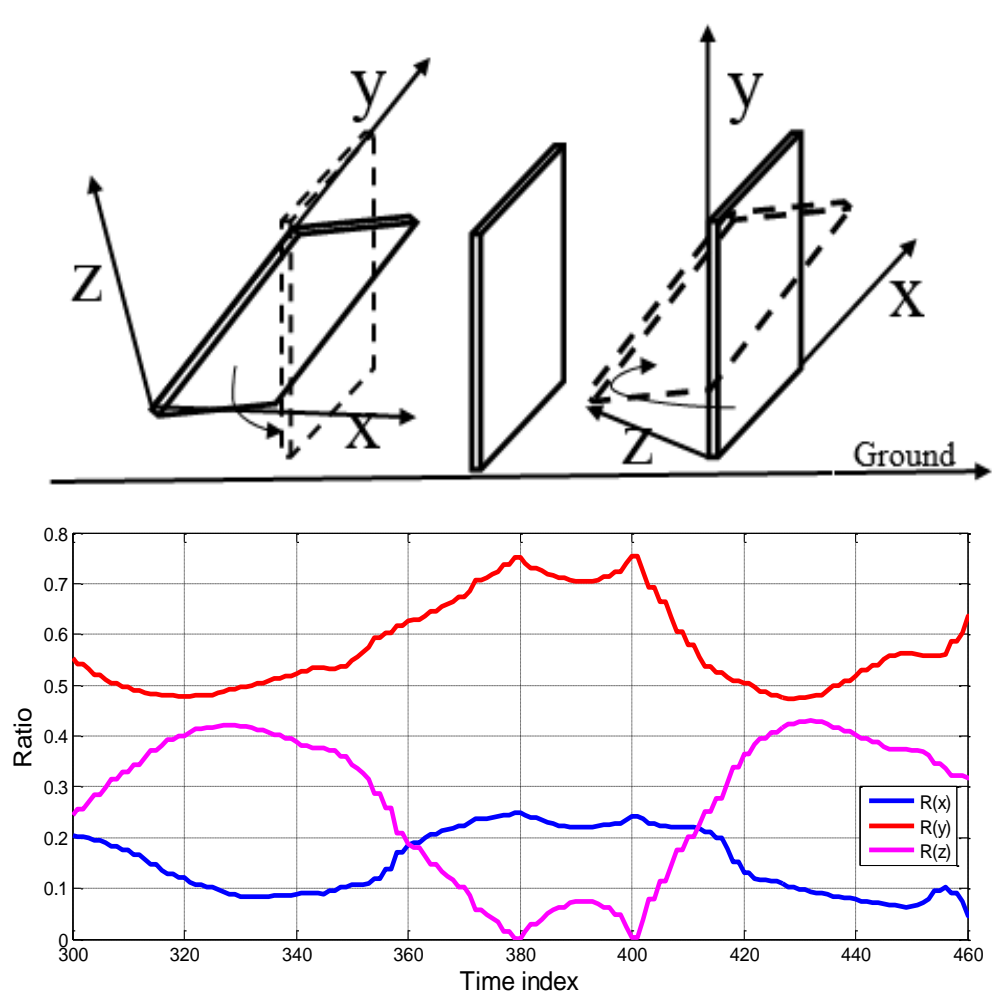

Figure 3. The Ratio Change when the Phone is Placed in the Trouser

\section{Pocket}

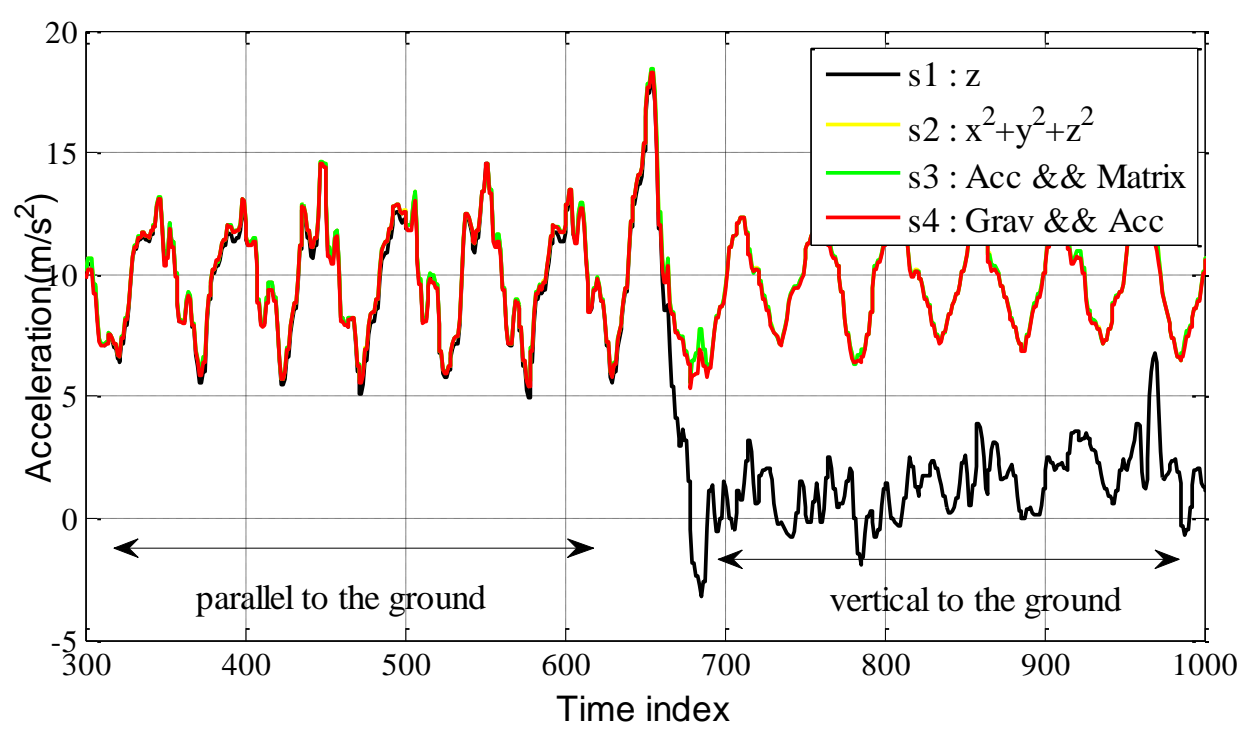

Figure 4. Changes of Acceleration Value when the User Walks

The effectiveness of the proposed scheme is demonstrated with experiments and the performance of the proposed scheme is compared with those of the conventional schemes. Figure 4 shows the changes of acceleration value in terms of various situations for considered schemes when the user walks. In the figure, the duration of time index from 300 to 650 represents the situation that the screen of smart-phone is 
parallel to the ground. The duration of time index from 700 to 1000 represents the situation that the screen of smart-phone faces to the face of the user and the y-axis is vertical to the ground. Four lines, s1, s2, s3, s4, represent four different methods respectively. As shown in the figure, when the orientation of the smart-phone is changed, the result of the conventional method 1 is unacceptable. It is shown that the orientation of the smart-phone has a great influence on the acceleration waveform and it is not a good way to rely on only one axis of the accelerometer.

\subsection{Low Pass Filter}

Figure 5 indicates the relationship between frequency and walking speed: as the increase of velocity, the frequency of step is increased. The speed that the legs move is measured in number of steps per minute (spm), often referred to as cadence. The average recreational runner has a cadence of about $150-170 \mathrm{spm}$, with variance due to factors such as individual height, level of general fitness. And it means that in our daily life, step frequency less than $4 \mathrm{~Hz}$.

In order to reduce of influence of high frequency noise, we need a low pass filter to process the raw acceleration sensor data. To filter out the noise, there is a scheme based on the frequency spectrum analysis and the examination of dominant frequencies using the fast Fourier transforms (FFT) [15]. Considering about time delay and the computational complexity, however, we decided to process the raw data in time domain. So, a low pass FIR filter of order 30 and with $4 \mathrm{~Hz}$ cutoff frequency is adopted in this paper.

For a causal discrete-time FIR filter of order N, each value of the output sequence is a weighted sum of the most recent input values:

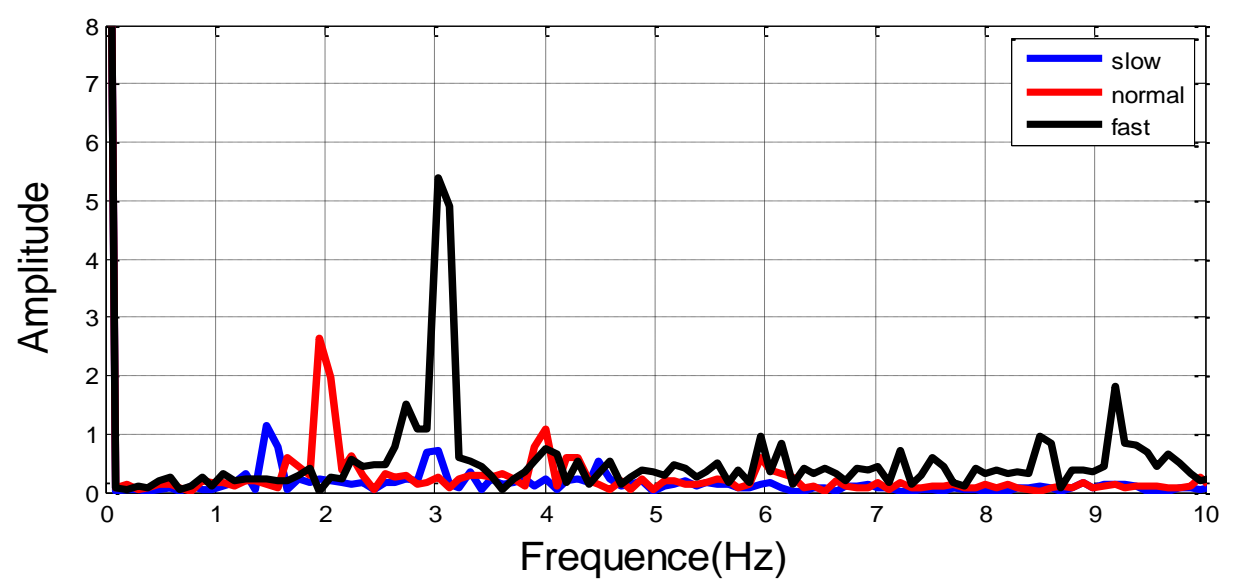

Figure 5. The Change of Frequency when Users Walking in Different Speed

$$
y(n)=\sum_{i=0}^{N} b_{i} x[n-i]
$$

where:

$\mathrm{x}[\mathrm{n}]$ is the input signal,

$\mathrm{y}[\mathrm{n}]$ is the output signal,

$\mathrm{N}$ is the filter order; an Nth-order filter has $(\mathrm{N}+1)$ terms on the right-hand side

$b_{i}$ is the value of the impulse response at the ith instant for $0 \leq \mathrm{i} \leq \mathrm{N}$ of an Nth-order

FIR filter. 


\subsection{Step Detection Rule}

An automatic peak detection scheme is introduced for a periodic and quasi-periodic signal [16]. If a new peak in the waveform of acceleration value is detected, it indicates that a new step has occurred. Therefore, the steps can be counted with the number of the peaks of waveform. However, the accuracy of conventional peak detection scheme is not good enough to be applied in our considered situations. To enhance the accuracy of the conventional scheme, a modified peak detection scheme is presented in this paper. To detect the peaks and the valleys, three adjacent data are stored in memory and the differences of the values of these three data are computed as follows:

$$
\begin{aligned}
& p_{n}=\mathrm{s}_{n-1}-\mathrm{s}_{n-2} \\
& p_{n}=\mathrm{s}_{n-1}-\mathrm{s}_{n}
\end{aligned}
$$

where $p_{n}$ is a flag that can be used for peak detection. If $p_{n-1}>0$ and $p_{n}>0, \mathrm{~s}_{\mathrm{n}-1}$ is a peak. If $p_{n-1}<0$ and $p_{n}<0, \mathrm{~s}_{\mathrm{n}-1}$ is a valley.

Unlike the conventional peak detection scheme using a threshold, two different thresholds are used for peak detection and valley detection, respectively. In this paper, for a convenience, either the peak or the valley is called as a pole point. In the proposed scheme, if two adjacent pole points are all peaks or valleys, the smaller one is abandoned. Only when the former pole point is peak and the following point is valley, there is a possibility that a new step is occurred. On the while, the time interval between two pole points is also considered importantly. If the time interval of two pole points is too short, the pole point also is ignored. The criteria of pole point detection as well as time interval are set through extensive experiments. The detailed flow chart of the proposed scheme is shown in Figure 6. 


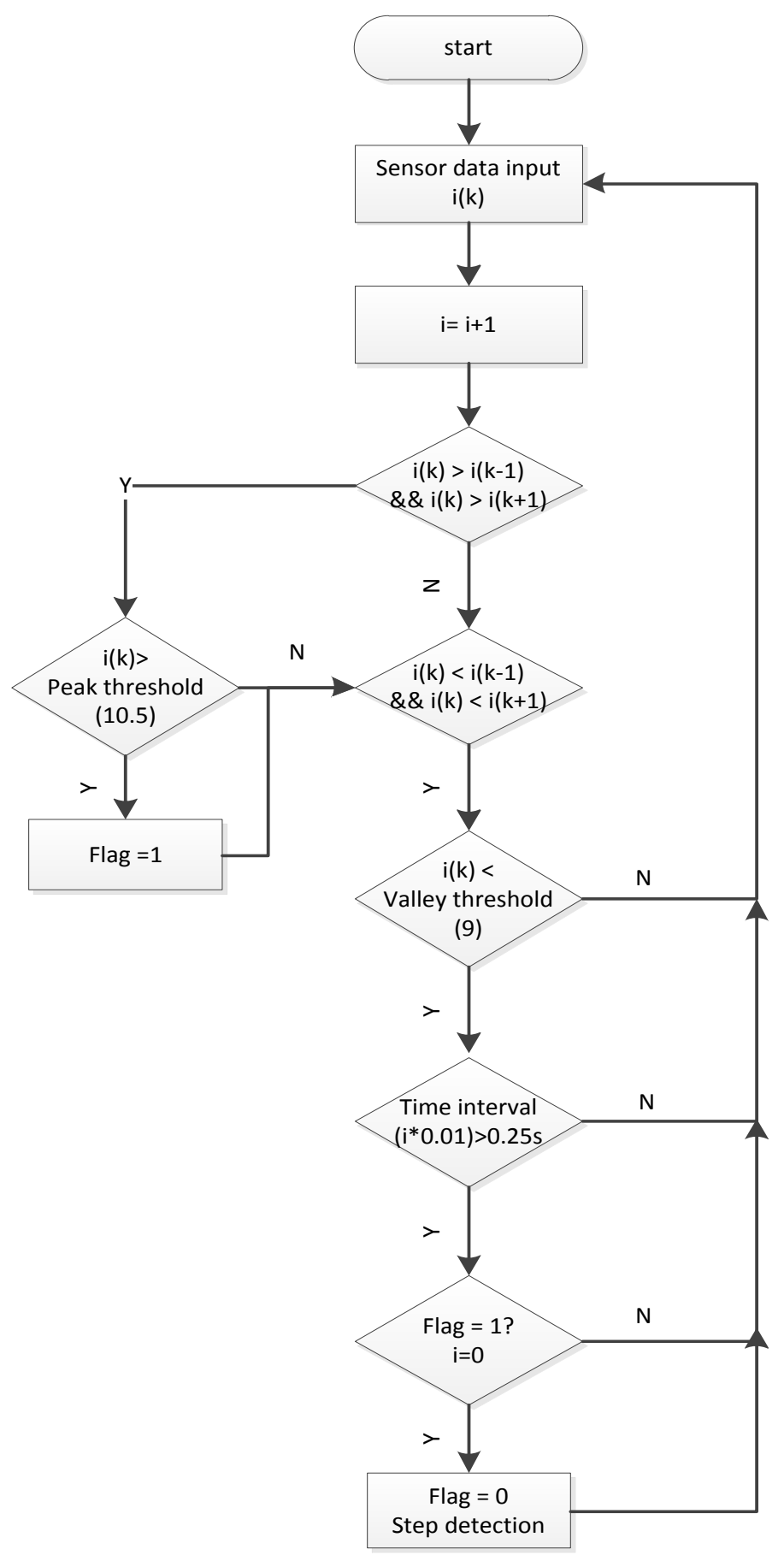

Figure 6. Flow chart of step detection

\section{Experimental Results}

To evaluate the performance of the proposed scheme, both walking and running cases were considered. Four situations, such as on hand, in handbag, in trouser pocket and in shirt pocket, were considered for the walking case, while two situations, on hand and in trouser pocket, were considered for running cases.

In the walking cases, 50 times of experiments were performed with 200 steps every time. Figure 7 shows the results of experiments. For the running case, 40 times of experiments were performed with 200 steps every time. Figure 8 shows the results of experiments. As shown in the figures, it is obvious that the proposed 
scheme provides higher accuracy and more stable performance compared to other conventional methods. Especially when the user runs, the performance of the proposed scheme based on the gravity and the acceleration sensors is a remarkably enhanced compared to other conventional methods. Note that the percentage is defined as the estimated steps over the real steps and the experiment index is the serial number of the experiments. The comparison of averaged estimation performance is summarized in Table 2. Meanwhile, in order to reflect the performance of this new schema, we download other top two step counting applications from Google Play Store and S-Health which is installed in the Samsung galaxy S4, and the final result of this test is shown in Table 1.

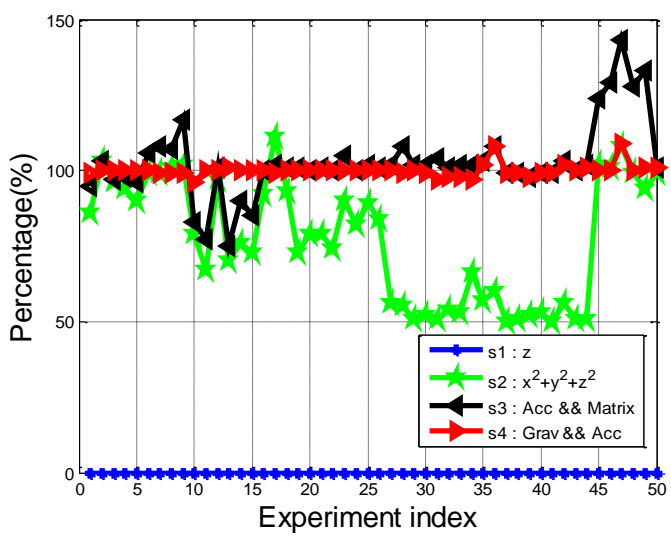

(a) trouser pocket

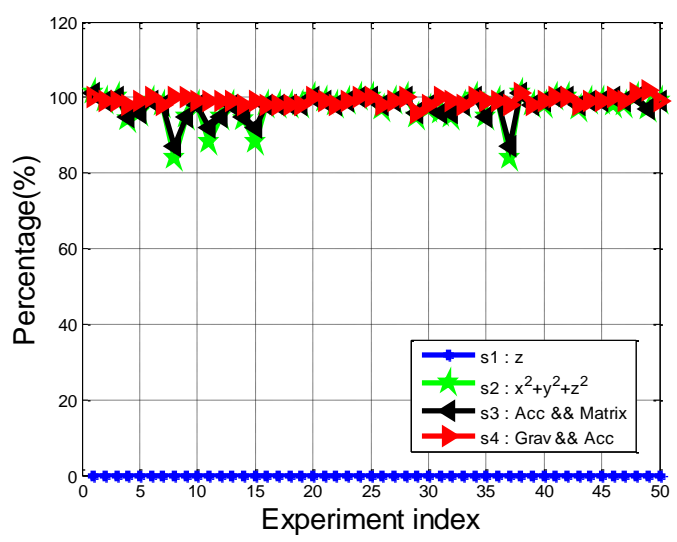

(c) shirt pocket

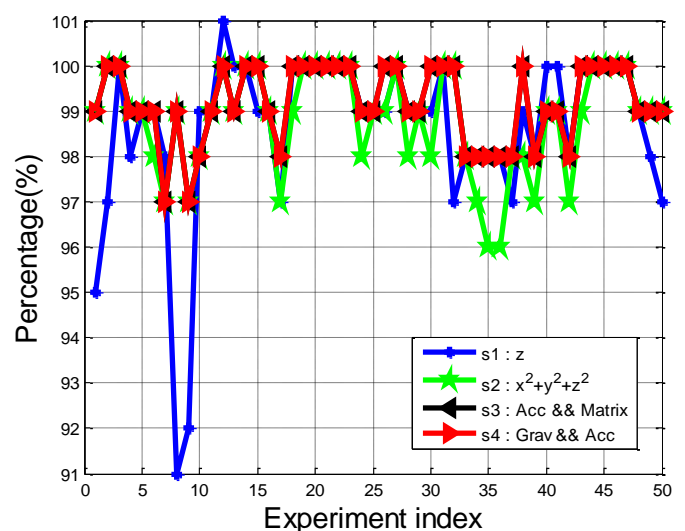

(b) on hand

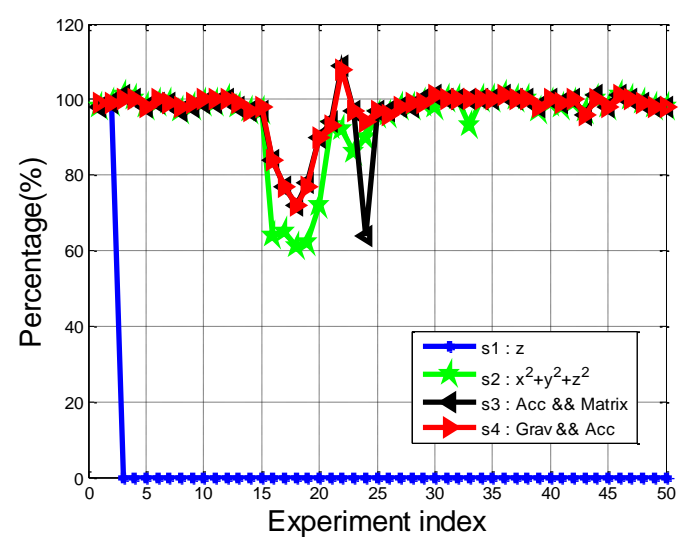

(d) hand bag

Figure 7. Experimental Results of Walking Cases 


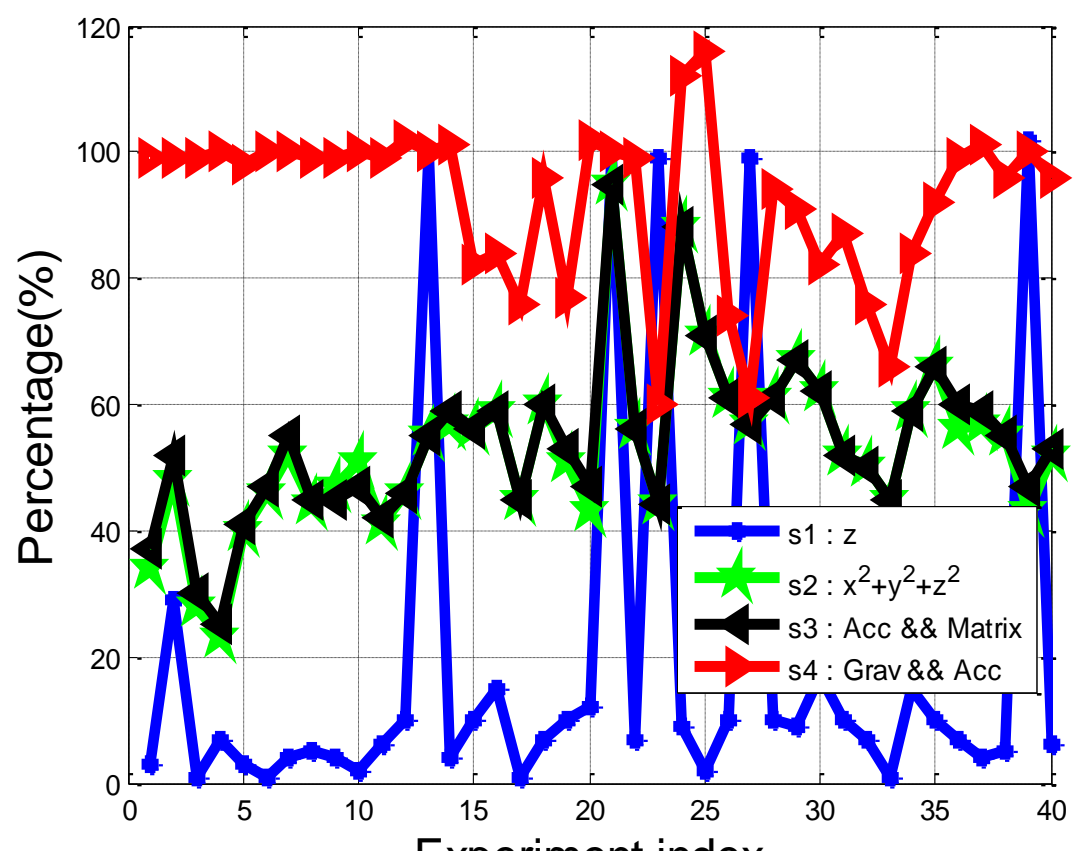

Experiment index

(a) on hand

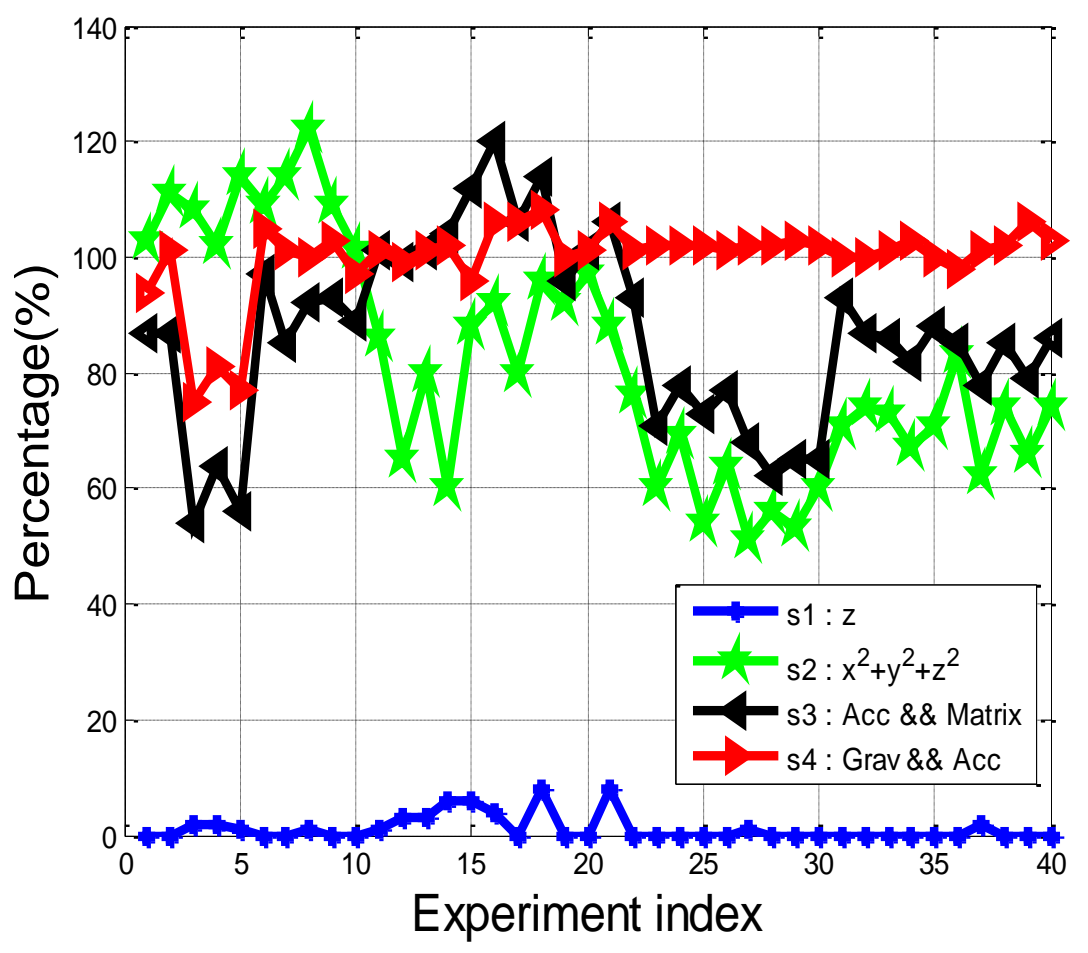

(b) trouser pocket

Figure 8. Experimental Results of Running Cases 
Table 1. The Performance of Different Applications

\begin{tabular}{|c|c|c|c|c|c|c|c|}
\hline & & 1 & 2 & 3 & 4 & 5 & Average \\
\hline \multirow{7}{*}{ hand } & our app & 100 & 101 & 100 & 100 & 101 & 100.4 \\
\cline { 2 - 8 } & S-Health & 99 & 99 & 99 & 100 & 100 & 99.4 \\
\cline { 2 - 8 } & Noom Walk & 100 & 99 & 99 & 100 & 100 & 99.6 \\
\cline { 2 - 8 } & $\begin{array}{c}\text { Pedometer } \\
\text { (Ver.1.3.7) }\end{array}$ & 97 & 97 & 97 & 98 & 99 & 97.6 \\
\hline \multirow{7}{*}{ pocket } & our app & 99 & 100 & 99 & 100 & 99 & 99.4 \\
\cline { 2 - 8 } & S-Health & 100 & 99 & 97 & 99 & 98 & 98.6 \\
\cline { 2 - 8 } & Noom Walk & 99 & 100 & 97 & 100 & 99 & 99.0 \\
\cline { 2 - 8 } & $\begin{array}{c}\text { Pedometer } \\
\text { (Ver.1.3.7) }\end{array}$ & 98 & 97 & 95 & 97 & 95 & 96.4 \\
\hline
\end{tabular}

Table 2. Comparison of Averaged Estimation Performance

\begin{tabular}{|c|c|c|c|c|c|c|}
\hline \multirow{2}{*}{ method } & \multicolumn{4}{|c|}{ walking } & \multicolumn{2}{c|}{ running } \\
\cline { 2 - 7 } & $\begin{array}{c}\text { trouser } \\
\text { pocket }\end{array}$ & hand & $\begin{array}{c}\text { shirt } \\
\text { pocket }\end{array}$ & handbag & $\begin{array}{c}\text { trouser } \\
\text { pocket }\end{array}$ & hand \\
\hline S1: $z$ & 0 & $98 \%$ & 0 & $13 \%$ & 0 & $19 \%$ \\
\hline S2: $\mathrm{x}^{2}+\mathrm{y}^{2}+\mathrm{z}^{2}$ & $77 \%$ & $99 \%$ & $97 \%$ & $94 \%$ & $81 \%$ & $53 \%$ \\
\hline S3: Acc\&Matrix & $97 \%$ & $99 \%$ & $97 \%$ & $96 \%$ & $87 \%$ & $54 \%$ \\
\hline S4: Grav\&Acc & $99 \%$ & $99 \%$ & $99 \%$ & $95 \%$ & $99 \%$ & $92 \%$ \\
\hline
\end{tabular}

\section{Conclusions}

In this paper, a novel step counting algorithm based on the built-in acceleration and gravity sensors of a smart-phone is proposed to enhance the estimation performance regardless of the position of a smart-phone and the motions of a pedestrian like walking or running. According to the results, the performance of the proposed scheme is enhanced compared to the conventional schemes no matter what the pedestrian is walking or running although the phone is in different places likewise trouser pocket, shirt pocket and hands. Therefore, the proposed scheme can be used for real-time indoor positioning system because it is more suitable for various practical situations.

\section{Acknowledgements}

This research was supported by the MSIP (Ministry of Science, ICT and Future Planning), Korea and NAVER Corp., under ICT/SW Creative research program supervised by the NIPA(National IT Industry Promotion Agency) (NIPA-2014-(H051114-1006)). 


\section{References}

[1] N. Chang, R. Rashidzadeh, M. Ahmadi. Robust indoor positioning using differential Wi-Fi access points. IEEE Transactions on Consumer Electronics. 56 (2010)

[2] H. Jeon, U. Jo, M. Jo, N. Kim, Y. Kim. An Adaptive AP Selection Scheme Based on RSS for Enhancing Positioning Accuracy. Wireless Personal Communications. 69 (2013)

[3] P. Brida, J. Machaj, J. Benikovsky. A Modular Localization System as a Positioning Service for Road Transport. Sensors. 14 (2014)

[4] J. Wang, D. Ni, K. Li. RFID-Based Vehicle Positioning and Its Applications in Connected Vehicles. Sensors. 14 (2014)

[5] K. Yun, Y. Jo, N. Kim, U. Jo, S. Yang, Y. Kim. A Practical Indoor Position Estimation by Using a Laptop Computer Equipped With Sensors. International Journal of Smart Home. 7, 4 (2013)

[6] J. Li, B. Zhang, H. Liu, L. Yu, Z. Wang. An Indoor Hybrid Localization Approach Based on Signal Propagation Model and Fingerprinting. International Journal of Smart Home. 7, 6 (2013)

[7] H. Su, S. Moh. Comparative Performance Study of Localization Schemes in Wireless Sensor Networks. International Journal of Smart Home. 8, 1 (2014)

[8] A.R. Jiménez, F. Seco, F. Zampella, J.C. Prieto, J. PDR with a Foot-Mounted IMU and Ramp Detection. Sensors. 11 (2011)

[9] S.H. Shin, M.S. Lee, C.G. Park. Pedestrian Dead Reckoning System with Phone Location Awareness Algorithm. IEEE/ION Position Location and Navigation Symposium (2010), May 4-6; Indian Wells, CA, USA.

[10] D. Gusenbauer, C. Isert, J. Krosche. Self-Contained Indoor Positioning on Off-the-Shelf Mobile Devices. International Conference on Indoor Positioning and Indoor Navigation (2010), Sept. 15-17; Zurich.

[11] S.H. Shin, C.G. Park. Adaptive step length estimation algorithm using optimal parameters and movement status awareness. Medical Engineering \& Physics. 33 (2011)

[12] S. Ayub, B.M. Heravi, A. Bahraminasab, B. Honary. Pedestrian Direction of Movement Determination using Smartphone. 6th International Conference on Next Generation Mobile Applications, Services and Technologies (2012), Sept. 12-14; Paris.

[13] S. Łuczak, W. Oleksiuk, M. Bodnicki. Sensing Tilt With MEMS Accelerometers. IEEE Sensors Journal. 6 (2006)

[14] I. Frosio, N. Alberto Borghese. Autocalibration of MEMS Accelerometers. IEEE Transactions on Instrumentation and Measurement. 58 (2009)

[15] A. Godfrey, R. Conway, D. Meagher, G. ÓLaighinb. Direct measurement of human movement by accelerometry. Medical Engineering \& Physics. 30 (2008)

[16] F. Scholkmann, J. Boss, M. Wolf. An Efficient Algorithm for Automatic Peak Detection in Noisy Periodic and Quasi-Periodic Signals. Algorithms. 5 (2012)

\section{Authors}

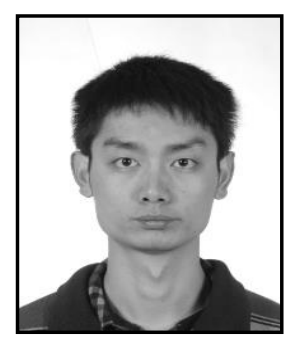

Qingchi Zeng, he received the B.S degree from Qingdao University of Science and Technology at Communication Engineering, Qingdao, China, in 2011. He is working toward the Master Degrees in the Advanced Wireless System and Technology Lab of Dept. of Electronic Engineering at Kwangwoon University, Seoul, Korea.

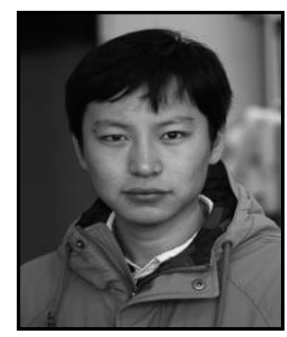

Biao Zhou, he received the B.S degree from Qingdao University of Science and Technology at Communication Engineering, Qingdao, China, in 2011. He is working toward the Master and Ph.D. Degrees in the Advanced Wireless System and Technology Lab of Dept. of Electronic Engineering at Kwangwoon University, Seoul, Korea. His research interests include ultra-wideband system, embedded system, and high accuracy positioning system. 


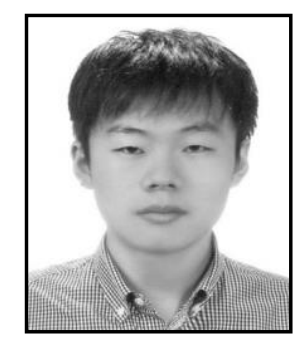

Changqiang Jing, he received the B.S. Degree from Qingdao University of Science and Technology at Communication Engineering, Qingdao, China, in 2008. He is working toward the Master and Ph.D. Degrees in the Advanced Wireless System and Technology Lab of Dept. of Electronic Engineering at Kwangwoon University, Seoul, Korea. His research interests include ultra-wideband system, embedded system, and high accuracy positioning system.

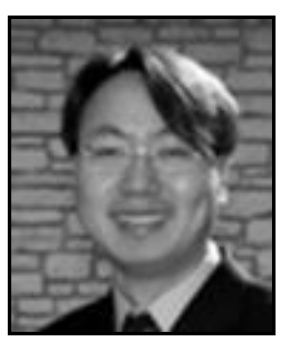

Youngok Kim, he received the B.S. degree in mechanical engineering from Yonsei University, Seoul, Korea in 1999, and the M.S. and Ph.D. degrees in electrical and computer engineering from the University of Texas at Austin, Austin, in 2002 and 2006, respectively. From 2006 to 2008, he was a senior researcher at Infra Laboratory of Korea Telecom (KT), Seoul, Korea. In March 2008, he joined the Department of Electronic Engineering of Kwangwoon University, Seoul, Korea, as a faculty member. His research interests include ultra-wide band wireless communication systems, OFDMbased systems, precise ranging and location systems, PAPR reduction techniques, diversity techniques for wireless systems, and multipleaccess schemes in multicarrier systems. 